\title{
Compensating Wage Differentials in Stable Job Matching Equilibrium
}

\author{
Seungjin Han* Shintaro Yamaguchi ${ }^{\dagger}$
}

January 17th, 2014

\begin{abstract}
This paper studies implicit pricing of non-wage job characteristics in the labor market using a two-sided matching model. It departs from the previous literature by allowing worker heterogeneity in productivity, which gives rise to a double transaction problem in a hedonic model. Deriving sufficient conditions under which assortative matching is the unique stable job-worker matching, we show that observed wage differentials between jobs reflect not only compensating wage differentials, but also worker productivity gaps between the jobs. We find that the job-worker matching pattern determines the extent to which compensating wage differentials are confounded with the worker productivity gap effect.
\end{abstract}

Keywords: hedonic model, heterogeneity, two-sided matching, matching pattern, wage differential, equalizing difference, worker productivity

JEL Codes: C78, J31

*Address: Department of Economics, McMaster University, Hamilton, Ontario Canada; Email: hansj@mcmaster.ca

${ }^{\dagger}$ Address: Department of Economics, McMaster University, Hamilton, Ontario Canada; Email: yamtaro@mcmaster.ca 


\section{Introduction}

The theory of equalizing differences has remained one of the most fundamental value theories in economics since Adam Smith's classic discussion in "The Wealth of Nations." Rosen (1974) develops a theory of equalizing differences for a commodity market and shows that implicit markets arise for differentiated products in which hedonic prices adjust so that all markets clear: The observed price differentials reflect the buyer's willingness to pay for a better product. ${ }^{1}$ The key idea of equalizing differences also holds in the labor market. Rosen (1986) applies the theory to the labor market and finds that equilibrium wage differentials reflect workers' willingness to pay for job characteristics. His theoretical finding demonstrates how non-wage characteristics of a job are valued and how workers are matched to such jobs.

Although the theory is a rich description of the labor market for heterogeneous jobs, it assumes homogeneous worker productivity. Rosen (1986) notes the importance of workers' productivity heterogeneity, pointing out

"...On the theoretical side of these questions, much more attention must

be paid to the value of workers' productivity characteristics and the nature of sorting and selection in those dimensions...."

The objective of this paper is to develop a theory of the labor market in which both job characteristics and worker productivity are heterogeneous. Our main question is how labor productivity and job characteristic are valued in the labor market. The difficulty in examining this problem arises from double transactions: workers sell their productivity characteristics to the firms, while the firms implicitly sell job characteristics to the workers. Double transactions are inherent in labor markets and absent in commodity markets. On the one hand, in the commodity market, consumers do not sell their characteristics to firms, and hence their characteristics do not directly affect firms' profits. On the other hand, in the labor market, not only

\footnotetext{
${ }^{1}$ The theory of equalizing differences has been applied to various types of commodity markets. For example, Scotchmer (1985) develops a hedonic price model in the housing market, and Kanemoto (1988) applies the theory to study the benefits of public projects. The theory is further developed by Ekeland (2010) and Chiappori et al. (2010) who undertake hedonic equilibrium analysis in a more general matching model.
} 
does the wage affect the employer's profit, but so do workers' characteristics. This implies that the wage difference between jobs reflects not only the compensating wage differentials, but also the difference in the productivity of the workers occupying the jobs. The presence of double transactions complicates our problem and prevents us from applying the known results for commodity markets.

Another question that arises is how heterogeneous jobs and workers are matched in the labor market. The double transaction issue also complicates the matching mechanism, since not only do worker's productivity characteristics affect a firm's profit, but the job characteristic in a firm affects worker's utility as well. A match is formed only when a firm and a worker agree on the wage that reflects the net value of the characteristics on both sides. The analysis is even harder when utility is not transferable, because the marginal utility of the job characteristic depends on the wage. The established results (Teulings 1995, Shimer 2005) in the optimal assignment literature for heterogeneous jobs and workers do not answer this question, because job characteristics do not affect the utility of a worker ${ }^{2}$, and hence, double transactions do not exist in those models.

In our model workers are heterogeneous in their productivity and jobs differ in their characteristics. Workers derive utility not only from wages, but also from the characteristics of the job they hold. The labor market is frictionless so that all workers can freely choose any job and all firms can freely hire any worker. We find that positive assortative matching is the unique stable matching between jobs and workers, if (i) a good job characteristic increases the marginal utility of consumption or the marginal productivity of a worker and (ii) the worker's utility function is concave in consumption. This result holds even when utility is not transferable.

We then show that the wage difference between two jobs reflects a compensating wage differential and the difference in productivity between workers occupying those two jobs. On the one hand, the former lowers the wage of a better job, because a worker is willing to accept a lower wage in exchange for better job characteristics. On the other hand, the latter raises the wage of a better job in assortative matching, because the worker occupying the better job is more productive. When the worker

\footnotetext{
${ }^{2}$ Sattinger (1975) also considers the matching problem, but the worker's utility function is not modeled.
} 
productivity effect dominates the compensating wage differentials, the observed wage gaps across jobs seem contradictory to the theory of equalizing differences, because the observed wage increases, rather than decreases, with job characteristics. We clearly characterize how this worker productivity effect masks compensating wage differentials in the data, and find that the job-worker matching pattern determines the extent to which the worker productivity effect appears in the wage. We further show that the distributions of jobs and workers pin down the job-worker matching pattern.

Empirical research often fails to find the evidence for compensating wage differentials. Various econometric reasons have been posited for the difficulty in estimating equalizing differences in the labor market. Among them, unobserved heterogeneity (Hwang et al. 1992), omitted variables (Brown 1980, Lucas 1977), measurement errors (Duncan and Holmlund 1983) and an unappealing linear approximation (Ekeland et al. 2004), have all been considered as sources of the counterintuitive results. All of these papers improve on the econometric methods, but are based on the model by Rosen (1986) in which worker productivity is homogeneous.

An explanation based on economic theory, rather than econometric theory, is provided by Hwang et al. (1998) and Lang and Majumdar (2004). They show that labor market frictions make it less likely to observe compensating wage differentials, using a search model with homogeneous worker productivity. Our model differs in that we allow for heterogeneous worker productivity and consider a frictionless economy. Our explanation is alternative to theirs and emphasizes the jobworker matching pattern as the main cause preventing us from interpreting the wage differences between jobs as compensating wage differentials.

The rest of the paper is structured as follows. We begin by reviewing the theory of equalizing differences in a labor market composed of homogeneous workers in Section 2. Then, we introduce heterogeneous worker productivity in Section 3. We derive sufficient conditions for assortative matching and characterize a stable equilibrium by the wage and matching functions. Section 4 discusses empirical implications of the main theoretical result. Section 5 concludes the paper. Proofs and technical discussions are collected in the Appendix. 


\section{Equalizing Differences}

To provide the intuition for equalizing differences, we begin with the analysis of the relationship between wage and job characteristics when labor productivity is homogeneous. Throughout the paper, consider a market with a continuum of firms and workers, each with the total measure of one. Firms differ in the characteristics of their jobs: firm $x$ has a job with characteristic $x \geq \underline{x}$, where $\underline{x} \in \mathbb{R}$ is the lowest job characteristic. Let $H(x)$ be the measure of firms whose job characteristics are no more than $x$.

For now, assume that workers are homogeneous in their productivity characteristic. Let the utility function $u(c, x)$ represent worker's preferences over the consumption good $c$ and the characteristic $x$ of her job. Worker's preferences are monotone, so that the marginal utilities $u_{c}(c, x)$ and $u_{x}(c, x)$ of the consumption good and the job characteristic respectively, are both positive for all $(c, x) \in \mathbb{R}_{+} \times \mathbb{R}$. Let workers' exogenous unearned income be normalized to zero. Then, if a worker's wage is $w$, she purchases $w$ units of the consumption good $(c=w)$.

The production function is given by $f(x, y)$, where $y$ is a worker's productivity characteristic. Because workers are homogeneous in their productivity, it can be rewritten as $f(x, \bar{y})$ where $\bar{y}$ is the same productivity for all workers. Let $w(x)$ be the equilibrium wage paid to the worker who takes the job with characteristic $x$. Because workers are homogeneous in their productivity, all of them must have the same equilibrium utility, $u(w(x), x)=\bar{u}$. Worker utility is increasing in job characteristic, so the less preferred job must be compensated by a higher wage in equilibrium in order to equalize the workers' utilities, i.e., $w(x)>w\left(x^{\prime}\right)$ if $x<x^{\prime}$.

Therefore, the observed wage should be negatively related to the job characteristic. This is recognized as an equalizing difference in the labor market or a compensating wage differential (see Rosen (1986).) This relationship is solely driven by the workers' monotonic preferences. It does not depend on the nature of the job characteristic in the production technology or on other properties of the workers' preferences. 


\section{Stable Matching Equilibrium}

\subsection{Environment}

We turn to the analysis of the labor market in which workers' productivity characteristics are heterogeneous. Heterogeneity in labor productivity gives rise to the double transaction problem as the worker sells her productivity characteristic to the firm, and the firm sells the job's characteristic to the worker. Therefore, the equilibrium wage reflects two prices: one for the worker's productivity characteristic and the other for the job characteristic. We show how these two prices determine the equilibrium wages by constructing a stable matching equilibrium where no alternative pairs of firms and workers can be strictly better off by matching and transferring wage.

Let $y \geq \underline{y}$ denote a worker's productivity characteristic, where $\underline{y} \in \mathbb{R}$ is the lowest productivity characteristic. Let $G(y)$ be the measure of workers whose productivity characteristics are no more than $y$. Recall that firm $x$ has a job with characteristic $x \geq \underline{x}$, and analogously $H(x)$ is the measure of firms whose job characteristics are no more than $x$. Denote by $h$ and $g$ the density functions for the distributions of the job characteristic and the worker's productivity, $H$ and $G$, respectively. For technical purposes, the values of densities are finite positive real numbers: $0<h(x)<\infty$ for all $x$ and $0<g(y)<\infty$ for all $y$. Let $f(x, y)$ denote a firm's production function. The production function is increasing in $y: f_{y}(x, y)>0$ for all values of $(x, y)$, which naturally follows from what productivity means. We do not impose a restriction on the sign of $f_{x}(x, y)$ : it can be either negative, zero, or positive. If necessary, we assume that utility and production functions are twice differentiable.

\subsection{Definition of Equilibrium}

A stable matching can be characterized by a matching function and a wage function. Let $m(x)$ be a matching function that specifies the productivity characteristic of the worker who works for firm $x$ in equilibrium. Let $w(x)$ be a wage function that 
specifies the wage firm $x$ pays to its worker. ${ }^{3}$

A worker's objective is to maximize her utility by deciding who to work for and at what wage level. In doing so, she must allow her employer to earn the profit at least as high as the potential profit the employer would earn given the matching and wage functions; in other words, $f(x, y)-w \geq f(x, m(x))-w(x)$. The worker's problem is formulated as (W1) below:

$$
\begin{gathered}
\max _{(x, w)} u(w, x) \\
\text { subject to } f(x, y)-w \geq f(x, m(x))-w(x) .
\end{gathered}
$$

If the constraint slacks, the worker can increase her utility by raising the wage $w$ without violating the constraint. Therefore, the constraint is binding at a solution to (W1).

A firm's objective is to maximize its profit by deciding whom to hire and at what wage level. In doing so, the firm must secure its employee the level of utility at least as high as the potential utility level that the employee would enjoy given the matching and wage functions. Let $n(y)$ be the job characteristic that worker $y$ is matched under $m(\cdot)$, i.e., $m(n(y))=y$. The firm's problem is formulated as (F1) below:

$$
\begin{gathered}
\max _{(y, w)}\{f(x, y)-w\} \\
\text { subject to } u(w, x) \geq u(w(n(y)), n(y)) .
\end{gathered}
$$

Similar to the worker's problem, the constraint in the firm's problem is also binding at a solution.

In a stable matching equilibrium, the solutions to problems (W1) and (F1) coincide with the matching function $y=m(x)$ and the wage function $w(x)$, and no pairs of firms and workers can make themselves strictly better off by matching and transferring wage.

Definition 3.1 For the given distributions of the job characteristics and workers' productivity characteristics, a stable matching equilibrium is given by a set of

\footnotetext{
${ }^{3}$ Note that the wage does not depend on $y$ given $x$ and the matching function, because $w(x, y)=$ $w(x, m(x))=w(x)$.
} 
matching and wage functions $\{m(\cdot), w(\cdot)\}$, if $(i)(x, w(x))$ is a solution to the problem (W1) for every worker $y$; (ii) $(y, w(x))$ is a solution to the problem (F1) for every firm $x$; (iii) $y=m(x)$; and (iv) the payoffs determined by $\{m(\cdot), w(\cdot)\}$ are equal to or greater than their reservation payoffs to remain unmatched for every worker $y$ and firm $x$.

\subsection{Assortative Matching}

In this subsection, we derive a sufficient condition for the matching function $m(\cdot)$ to exhibit a positive assortative matching in which high productivity workers are matched to firms with better jobs. We begin by showing that the worker's equilibrium utility is increasing in her productivity. This lemma holds in any stable matching equilibrium.

Lemma 3.1 In a stable matching equilibrium characterized by $\{m(\cdot), w(\cdot)\}$, the worker's equilibrium utility is increasing in her productivity characteristic $y$.

Proof. See Appendix 6.1.

This result comes from the monotonicity of worker preferences and the monotonicity of the production function in the worker's productivity characteristic.

When workers are homogeneous, a less preferred job characteristic must be compensated by a higher wage in order to equalize the equilibrium utility across workers as shown in Section 2. However, when workers' productivity is heterogeneous, the relationship between the equilibrium wage and the job characteristic is not that obvious, because various combinations of wages and job characteristics can increase worker's utility. This is how the double transaction problem complicates the analysis of the equilibrium wage. The increasing property of the equilibrium utility leads to the following lemma.

Lemma 3.2 Let $\underline{w}(x, u)$ be the lowest wage for firm $x$ to maintain the worker's utility level at $u$. If the utility function is concave in consumption (i.e., $u_{c c}(c, x) \leq 0$ ) and supermodular (i.e., $u_{c x}(w, x) \geq 0$ ), the following inequality holds:

$$
\underline{w}\left(x_{L}, u_{H}\right)-\underline{w}\left(x_{L}, u_{L}\right) \geq \underline{w}\left(x_{H}, u_{H}\right)-\underline{w}\left(x_{H}, u_{L}\right),
$$


where the strict inequality holds if the utility function is strictly supermodular (i.e., $\left.u_{c x}(w, x)>0\right)$.

\section{Proof. See Appendix 6.2.}

Consider two firms $x_{H}$ and $x_{L}\left(x_{H}>x_{L}\right)$ that attempt to hire one of the two workers $y_{H}$ and $y_{L}\left(y_{H}>y_{L}\right)$. Each firm must offer a higher wage to the $y_{H}$ worker , because the $y_{H}$ worker receives a higher utility than the $y_{L}$ worker in equilibrium (see Lemma 3.1). Equation (3.1) shows that the difference in the firm $x_{L}$ 's wage offers between the two workers is at least as large as the difference in the firm $x_{H}$ 's. This result is used to prove the following theorem. Note that many commonly used production and utility functions satisfy supermodularity. ${ }^{4}$

Theorem 3.1 The matching function $m(\cdot)$ is increasing if the utility function is concave in consumption (i.e., $u_{c c}(c, x) \leq 0$ at each $(c, x)$ ) and Condition 1 or 2 is satisfied:

1. $u(\cdot, \cdot)$ is supermodular and $f(\cdot, \cdot)$ is strictly supermodular

2. $u(\cdot, \cdot)$ is strictly supermodular and $f(\cdot, \cdot)$ is supermodular

\section{Proof. See Appendix 6.3.}

Assortative matching under transferable utility with a strict supermodular production function is well-known. ${ }^{5}$ Suppose that the worker's utility function is quasilinear in wage so that it is given by $u(w, x)=v(x)+w$, where $v(\cdot)$ is an increasing and concave function. This assumption implies transferable utility, because one unit of the profit $f(x, y)-w$ can be transferred to the worker as one unit of utility. In

\footnotetext{
${ }^{4}$ In our context, the supermodular utility functions include, among many others, (a) substitutable consumption and job characteristic: $u(c, x)=\alpha c^{a}+\beta x^{b}$ with $\alpha \geq 0$ and $\beta \geq 0$ (b) perfect complements: $u(c, x)=\min \{\alpha c, \beta x\}$ with $\alpha \geq 0$ and $\beta \geq 0$. (c) Cobb-Douglas utility function: $u(c, x)=\beta c^{t} x^{s}$ with $\beta \geq 0, t \geq 0, s \geq 0$, and (d) constant elasticity of substitution utility function: $u(c, x)=\left(\alpha c^{\rho}+\beta x^{\rho}\right)^{1 / \rho}$ with $\alpha \geq 0, \beta \geq 0, \rho \leq 1$. The classes of utility functions, (c) and (d), are strictly supermodular if the parameter restrictions hold with strict inequality. The examples of the common supermodular production functions are similar.

${ }^{5}$ The first contribution is Becker (1973). Also see Topkis (1998) for more details.
} 
this case, a strict supermodular production function ensures assortative matching. Condition 1 of theorem 3.1 includes this case.

Theorem 3.1 generalizes the well-known result to the case of non-transferable utility as well. Suppose that the worker's utility function is not separable so that it is given by $u(w, x)$. This generalization allows us to consider the case in which the job characteristic affects the marginal utility of consumption (i.e. wage). Under this preference, one unit of wage is not transferable to the worker as one unit of utility. Condition 2 of theorem 3.1 includes this case. Theorem 3.1 shows that assortative matching arises even if utility is not transferable and the production function is not strictly supermodular as long as Condition 2 is met.

Legros and Newman (2007) also derive a sufficient condition for assortative matching when utility is not transferable. One difference in our paper is that we consider the case in which continuum of agents exist, while Legros and Newman (2007) consider the model in which finite number of agents exist. Thanks to the continuity, we can clearly characterize the relationship between wage differentials and the matching pattern in terms of a differential equation, as seen in the next subsection.

Another difference is that we present the sufficient condition in terms of properties of the primitive payoff functions themselves, while Legros and Newman (2007) provide it in terms of property of the function that specifies the agent's maximum payoff conditional on the payoff for its matching partner, which is called Generalized Increasing Differences (GID). ${ }^{6}$

Our result allows one to readily examine not only whether the condition is satisfied or not, but also whether the condition is economically plausible or not. For example, similar to Theorem 3.1, we can establish the sufficient condition for neg-

\footnotetext{
${ }^{6}$ We can show that the primitive payoff function satisfying our sufficient condition also has the strict GID property. Both the sufficient condition with continuum of agents in Theorem 3.1 and Legros and Newman's GID with finite number of agents presume the existence of a stable matching equilibrium. Therefore, one can establish the assortative matching for continuum of agents by applying GID, if she can also show the existence of stable matching equilibrium using the function that specifies agent's maxium payoff conditional on the matching partner's payoff. However, such approach does not enable the intuitive characterization of the relationship between wage differentials and the matching pattern as we will show in Section 3.4. This is why we establish the sufficient condition and prove the existence of stable matching equilibrium (see Section 3.5) using the primitive payoff functions.
} 
atively assortative matching by replacing the concavity assumption of the worker's utility function with the convexity and the supermodular assumption with the submodular one. However, the convexity assumption seems less intuitive, which is why we focus on positive assortative matching in this paper.

\subsection{Equilibrium Characterization}

In this subsection, we show the necessary and sufficient condition for the wage and matching functions to characterize a stable matching equilibrium.

Proposition 1 Suppose that the utility function is concave in consumption and Condition 1 or 2 in Theorem 3.1 is satisfied. Then, the matching and wage functions $\{m(\cdot), w(\cdot)\}$ characterize a stable matching equilibrium if and only if:

1. For all workers and firms, the matching and wage functions $\{m(\cdot), w(\cdot)\}$ provide payoffs equal to or higher than their reservation payoffs to remain unmatched.

2. $\forall x \geq \underline{x}$,

$$
H(x)=G(m(x))
$$

3. $\forall x \geq x$,

$$
w^{\prime}(x)+\frac{u_{x}(w(x), x)}{u_{c}(w(x), x)}=f_{y}(x, m(x)) m^{\prime}(x)
$$

Proof. See Appendix 6.4.

Equation (3.2) characterizes the assortative matching function $m(\cdot)$ as the unique stable matching function. Equation (3.3) is the first-order necessary condition for the wage function that characterizes solutions for both firm's problem (F1) and worker's problem (W1). If Conditions 2 and 3 are satisfied, no pairs of a firm and a worker can make themselves strictly better off by matching and transferring wage.

Equation (3.3) clearly shows the relationship between job characteristics, worker productivity, and wages in a stable matching equilibrium. The right-hand side of Equation (3.3) shows that, as the job characteristic increases at the margin, the 
productivity of the worker occupying the job also increases by $m^{\prime}(x)$, which consequently results in a change in the total output by $f_{y}(x, m(x)) m^{\prime}(x)$. In a competitive labor market, this change in the total output is fully passed onto the worker. On the left-hand side of Equation (3.3), the first term is the marginal change of wage, and the second term is the marginal change in the worker's utility due to the change in job characteristics normalized by the marginal utility of wage. Hence, this second term is the dollar value of the marginal utility of the job characteristics, which is precisely the compensating wage differential.

\subsection{Existence and Uniqueness of Equilibrium}

Note that Equation (3.3) is a first-order differential equation for the wage function $w(\cdot)$ given the assortative matching function characterized in Condition 2 in Proposition 1:

$$
w^{\prime}(x)=\phi(x, w(x))
$$

where the function $\phi(\cdot, \cdot)$ is defined as

$$
\phi(x, w(x)):=-\frac{u_{x}(w(x), x)}{u_{c}(w(x), x)}+f_{y}(x, m(x)) m^{\prime}(x) .
$$

Hence, the wage function can be derived by solving this first-order differential equation with an initial condition of the wage $w(\underline{x})$ in the bottom match. The exact level of the wage in the bottom match will be ultimately determined by bargaining power each side has, which may depend on various factors, such as legal and cultural environment as well as which side of the market has more potential entrants. The existence and uniqueness of an equilibrium come down to the existence and uniqueness of a solution to the first-order differential equation (3.4). For the existence and uniqueness, consider the following conditions.

Condition 1. $f_{y}(x, m(x)) m^{\prime}(x)$ is continuous in $x$.

Condition 2. $\frac{u_{x}(w, x)}{u_{c}(w, x)}$ is (i) continuous in $x$ and (ii) Lipschitz continuous in $w$, 
that is, there exists a scalar $0 \leq k<\infty$ such that, for each $x$ and all $w, w^{\prime}$,

$$
\left|\frac{u_{x}(w, x)}{u_{c}(w, x)}-\frac{u_{x}\left(w^{\prime}, x\right)}{u_{c}\left(w^{\prime}, x\right)}\right| \leq k\left|w-w^{\prime}\right|
$$

If Conditions 1 and 2 are satisfied, then the function $\phi(\cdot, \cdot)$ is Lipschitz continuous in $w$ and continuous in $x$. This ensures a unique solution $w(x)$ given an initial condition $w(\underline{x})$ by the Picard-Lindelof Theorem (See Teschl (2012)).

The slope of the matching function is given by $m^{\prime}(x)=h(x) / g(m(x))$ by taking a derivative of both sides of Equation (3.2). Because the values of the densities $h$ and $g$ are assumed to be positive finite numbers, the value of $m^{\prime}(x)$ is a positive finite number. If we further assume that the densities are continuous, then $m^{\prime}(\cdot)$ is continuous as well. Condition 1 is then satisfied if $f_{y}(\cdot, m(\cdot))$ is continuous in $x$, which is satisfied if $f_{y}(\cdot, \cdot)$ is continuous in both $x$ and $y$ because $m(\cdot)$ is continuous.

As for Condition 2, note that $\frac{u_{x}(w, x)}{u_{c}(w, x)}$ is the marginal rate of substitution of the job characteristic for consumption. This is continuous in $x$ if each marginal utility function is continuous in $x$. The Lipschitz continuity with respect to $w$ specified in Equation (3.6) essentially requires that the marginal rate of substitution be not blown to infinity at any possible wage level given a job characteristic. Therefore, the ratio of the difference between the two marginal rates of substitution to the difference between the two corresponding wages is always bounded by some scalar $k$.

\subsection{Heterogeneous Worker Preferences}

The model can easily incorporate heterogeneity in worker preferences. Suppose that $N$ groups of workers exist. For example, a group of males and a group of females exist, with $N=2$ if males and females have different preferences over consumption and job characteristics. Let the preferences for workers in group $i$ be represented by the utility function $u^{i}(c, x)$. Denoting the wage and matching functions group $i$ by $w_{i}(x)$ and $m_{i}(x)$, respectively, the first-order condition (3.3) for group $i$ is given by

$$
w_{i}^{\prime}(x)+\frac{u_{x}^{i}(w(x), x)}{u_{c}^{i}(w(x), x)}=f_{y}\left(x, m_{i}(x)\right) m_{i}^{\prime}(x) \text { for } i=1, \ldots, N
$$


Finally, in equilibrium, firm $x$ is indifferent to the group from which it hires a worker. This condition implies that for all $x$,

$$
f\left(x, m_{i}(x)\right)-w_{i}(x)=f\left(x, m_{j}(x)\right)-w_{j}(x) \text { for all } i, j .
$$

\section{Empirical Implications}

In this subsection we discuss the empirical implications of Proposition 1. To see how the equilibrium wage varies with job characteristics, we rearrange Equation (3.3) as

$$
w^{\prime}(x)=-\frac{u_{x}(w(x), x)}{u_{c}(w(x), x)}+f_{y}(x, m(x)) m^{\prime}(x) .
$$

The first term on the right-hand side is the (negative) marginal rate of substitution of the job characteristic for consumption, and thus, compensating wage differentials. When workers are homogeneous in productivity, i.e., $m^{\prime}(x)=0$, wage differentials across jobs reflect only the compensating wage differentials, which confirms the theoretical prediction of equalizing differences. However, when workers are heterogeneous in productivity and they are assortatively matched with jobs, i.e., $m^{\prime}(x)>0$, the observed wage differentials across jobs $w^{\prime}(x)$ include the compensating wage differentials and the difference in worker productivity across jobs.

The key insight is that the slope of the matching function $m^{\prime}(x)$ determines the extent to which the difference in worker productivity affects the observed wage differentials across jobs $w^{\prime}(x)$. On the one hand, wages decrease with job characteristic $x$ for compensating wage differentials. On the other hand, wages increase with job characteristic for a higher worker productivity, when matching is positively assortative (i.e. $m^{\prime}(x)>0$ ). The observed wage differentials across jobs show the net effect of the two. Depending on the size of the slope of the matching function $m^{\prime}(x)$, compensating wage differentials may be completely offset by the worker productivity effect.

The shape of the matching function $m(x)$ is determined by the distributions of jobs $x$ and workers $y$. Recall that we denoted by $h$ and $g$ the density functions for 
the distributions of the job characteristic and the worker's productivity, $H$ and $G$, respectively. The assortative matching function $m(\cdot)$ is derived by Equation (3.2). Taking the derivatives of both sides of this equation yields

$$
m^{\prime}(x)=\frac{h(x)}{g(m(x))} .
$$

The slope of the matching function $m(x)$ at $x$ is the ratio of the density of the job characteristic $h(\cdot)$ to the density of the worker's productivity $g(\cdot)$. This ratio can be interpreted as a local measure of dispersion of worker productivity relative to that of job characteristics at $x$, since a lower density can be interpreted as more dispersion.

This point is clearer under a certain class of parametric distribution functions. When the distributions, $H$ and $G$, are in the class of Weibull, Pareto, Fréchet, Gumbel, or log-normal distribution, the logarithm of the matching function is given by

$$
\ln y=\ln m(x)=\ln k+q \ln x,
$$

where the parameter $q$ is the elasticity. In particular, when the distributions are lognormal, the elasticity is given by the ratio of the standard deviations $q=\sigma_{y} / \sigma_{x}$, where $\sigma_{x}$ and $\sigma_{y}$ are the standard deviations of worker productivity and job characteristics, respectively. This example clearly shows that the slope of the matching function is determined by the relative dispersion of worker productivity to job characteristics.

Our analysis here shows that the observed wage differentials across jobs do not necessarily reflect only compensating wage differentials. Moreover, we find that in economies where the relative dispersion of worker productivity is higher, we observe less of evidence for compensating wage differentials; we may even observe wages increasing with job characteristic. The driving factor of our result is the matching pattern of jobs and workers that is determined by their distributions. Hwang et al. (1998) and Lang and Majumdar (2004) also find that the observed wage differentials across jobs are not necessarily reflected solely by compensating wage differentials. However, their findings are significantly different from ours in that worker productivity is homogeneous and labor market friction plays the central 
role in their model.

\section{Conclusion}

Rosen (1986) emphasizes that much more attention must be paid to the value of workers' productivity and the nature of sorting and selection along that dimension in order to facilitate a thorough understanding of compensating wage differentials; an important point given that productivity is arguably the most important worker characteristic. The main analytical challenge this presents is the existence of the double transaction in the labor market. The presence of the double transaction prevents us from applying known results that are suitable to markets lacking a double transaction, such as commodity markets.

There are two main results in our paper. First, we show the condition under which an assortative matching of jobs and workers arises as the unique stable matching in a two-sided matching model with continuum of jobs and workers. Second, using the differential equation for the equilibrium wage and matching, we explicitly show how compensating wage differentials and worker productivity differences affect the wage structure across jobs. The key insight of the theory is that the job-worker matching pattern drives the extent to which the worker productivity effect appears in the wage.

We note two important directions for future research. The first one is to allow for more than one dimension in job and worker characteristics. While showing the existence of stable matching under this environment may not be too complicated, characterizing a stable matching pattern seems particularly difficult. Such characterization would uncover the intuition behind how each characteristic component is valued and how the valuation of each characteristic affects compensating wage differentials. The second direction for future research is to introduce labor market frictions. We conjecture that, even in the presence of search frictions, equilibrium wage differentials across jobs reflect the net effect of compensating wage differentials and worker productivity gap. However, search frictions may weaken the effect of worker productivity gap as well as the effect of compensating wage differentials, because workers and jobs are not matched in a fully assortative way under the 
presence of labor market frictions. We leave those problems for our future research.

\section{References}

[1] Becker, G. (1973): “A Theory of Marriage: Part I," Journal of Political Economy, 81(4), 813-846.

[2] Brown, C. (1980): "Equalizing differences in the labor market" Quarterly Journal of Economics, 94(1): 113-141.

[3] Chiappori, P. A., R. J. McCann, and L. Nesheim (2010): “Hedonic price equilibria, stable matching and optimal transport: equivalence, topology, and uniqueness," Economic Theory, 42(2): 317-354.

[4] Duncan G. J., and B. Holmlund (1983): "Was Adam Smith right after all? another test of the theory of compensating wage differentials," Journal of Labor Economics, 1(4): 366-379.

[5] Ekeland, I. (2010): "Existence, uniqueness and efficiency of equilibrium in hedonic markets with multidimensional types," Economic Theory, 42(2), 275-315.

[6] Ekeland, I., J. Heckman, and L. Nesheim (2004): "Identification and estimation of hedonic models," Journal of Political Economy, 112(1; P2): S60-109.

[7] Hwang, H., W. R. Reed, and C. Habbard (1992): "Compensating wage differentials and unobserved productivity," Journal of Political Economy, 100(4): 835-858.

[8] Hwang, H., D. T. Mortensen, and W. R. Reed (1998): "Hedonic wages and labor market search," Journal of Labor Economics, 16(4): 815-847.

[9] Kanemoto, Y. (1988): "Hedonic Prices and the Benefits of Public Projects," Econometrica, 56(4), 981-989. 
[10] Lang, K. and S. Majumdar (2004): "The pricing of job characteristics when markets do not clear: theory and policy implications," International Economic Review, 45(4): 1111-1128.

[11] Legros, P. and A. Newman (2007): "Beauty is a beast, frog is a prince: assortative matching with nontransferabilities," Econometrica, 75(4): 1073-1102.

[12] Lucas, R. (1977): "Hedonic wage equations and psychic wages in the returns to schooling," American Economic Review, 67(4): 549-558.

[13] Rosen, S. (1974): "Hedonic prices and implicit markets: Product differentiation in pure competition," Journal of Political Economy, 82(1): 34-55.

[14] Rosen, S. (1986): "The theory of equalizing differences," In Ashenfelter, O. and R. Layrad, editors, Handbook of Labor Economics. North-Holland, Amsterdam.

[15] Sattinger, M. (1977): "Compensating Wage Differences," Journal of Economic Theory, 16(2), 496-503.

[16] Scotchmer, S. (1985): "Hedonic Prices and Cost/Benefit Analysis," Journal of Economic Theory, 37, 55-75.

[17] Shimer, R. (2005): "The Assignment of Workers to Jobs in an Economy with Coordination Frictions," Journal of Political Economy, 113(5), 996-1025.

[18] Teschl, Gerald (2012): Ordinary Differential Equations and Dynamical Systems, American Mathematical Society, Graduate Studies in Mathematics, Volume 140.

[19] Teulings, C. N. (1995): "The Wage Distribution in a Model of the Assignment of Skills to Jobs," Journal of Political Economy, 103(2), 280-315. 
[20] Topkis, D. M. (1998): Supermodularity and Complementarity, Frontiers of Economic Research. Princeton, N.J.: Princeton University Press. 


\section{Proofs}

\subsection{Proof of Lemma 3.1}

Consider any two workers, worker $y_{H}$ and worker $y_{L}$ such that $y_{H}>y_{L}$. Remember that $n(y)$ is the job characteristic that worker $y$ is matched under $m(\cdot)$, i.e., $m(n(y))=y$. The equilibrium utility for worker $y$ is $u(w(n(y)), n(y))$. The highest wage that the firm $n\left(y_{L}\right)$ is willing to offer to the worker $y_{H}$ is

$$
\hat{w}_{L, H}=f\left(n\left(y_{L}\right), y_{H}\right)-f\left(n\left(y_{L}\right), y_{L}\right)+w\left(n\left(y_{L}\right)\right),
$$

because firm $n\left(y_{L}\right)$ must earn the profit as high as it can earn by hiring the worker $y_{L}$. Because the firm $n(y)$ hires a worker $y$ in a stable matching equilibrium, we have a no-deviation condition,

$$
u\left(w\left(n\left(y_{H}\right)\right), n\left(y_{H}\right)\right) \geq u\left(\hat{w}_{L, H}, n\left(y_{L}\right)\right),
$$

where the left-hand side is the equilibrium of utility of worker $y_{H}$, and the righthand side is the highest utility that the firm $n\left(y_{L}\right)$ can give to the worker $y_{H}$.

Firm $n\left(y_{L}\right)$ produces more when it is matched with worker $y_{H}$ than worker $y_{L}$, implying that $f\left(n\left(y_{L}\right), y_{H}\right)-f\left(n\left(y_{L}\right), y_{L}\right)>0$. This means that the highest possible wage $\hat{w}_{L, H}$ that firm $n\left(y_{L}\right)$ offers worker $y_{H}$ is strictly higher than the equilibrium wage $w\left(n\left(y_{L}\right)\right)$ that the same firm offers worker $y_{L}$ (see Equation 6.1), implying that $\hat{w}_{L, H}>w\left(n\left(y_{L}\right)\right)$. Hence, we have the following inequality,

$$
u\left(\hat{w}_{L, H}, n\left(y_{L}\right)\right)>u\left(w\left(n\left(y_{L}\right)\right), n\left(y_{L}\right)\right) .
$$

Combining inequalities (6.2) and (6.3), we have

$$
u\left(w\left(n\left(y_{H}\right)\right), n\left(y_{H}\right)\right)>u\left(w\left(n\left(y_{L}\right)\right), n\left(y_{L}\right)\right),
$$


where the left-hand side is the equilibrium utility of worker $y_{H}$ and the right-hand side is the equilibrium utility of worker $y_{L}$. This proves that a worker's equilibrium utility is increasing in her characteristic.

\subsection{Proof of Lemma 3.2}

The lowest wage function $\underline{w}(x, u)$ can implicitly be defined by the equation $u(\underline{w}(x, u), x)-$ $u=0$. Taking the cross partial derivative of $u(\underline{w}(x, u), x)-u=0$ with respect to $x$ and $u$ yields

$$
\underline{w}_{x u}(x, u)=-\frac{u_{c c}(w, x)}{u_{c}(w, x)} \underline{w}_{x}(x, u) \underline{w}_{u}(x, u)-\frac{u_{c x}(w, x)}{u_{c}(w, x)} \underline{w}_{u}(x, u) .
$$

Given the utility level $u$, if the job characteristic increases, the wage must decrease: $\underline{w}_{x}(x, u)<0$. If the utility level $u$ increases given the job characteristic, the wage must increase: $\underline{w}_{u}(x, u)>0$. Because the worker's utility function is increasing in consumption, we have $u_{c}(w, x)>0$. Given the signs of the above derivatives and the concavity $\left(u_{c c}(c, x) \leq 0\right)$ and supermodularity $\left(u_{c x}(w, x) \geq 0\right)$ assumptions, we have $\underline{w}_{x u}(x, u) \leq 0$ at each $(x, u)$.

Consider a pair of utility levels $\left(u_{H}, u_{L}\right)$ where $u_{H}>u_{L}$ and a pair of job characteristics $\left(x_{H}, x_{L}\right)$ where $x_{H}>x_{L}$. The non-positive sign of $\underline{w}_{x u}(x, u)$ is equivalent to

$$
\underline{w}\left(x_{L}, u_{H}\right)-\underline{w}\left(x_{L}, u_{L}\right) \geq \underline{w}\left(x_{H}, u_{H}\right)-\underline{w}\left(x_{H}, u_{L}\right) .
$$

If the worker's utility function is strictly supermodular (i.e., $\left.u_{c x}(w, x)>0\right), \underline{w}_{x u}(x, u)$ is strictly negative, and hence, Equation (6.5) holds with strict inequality.

\subsection{Proof of Theorem 3.1.}

Suppose instead that the matching function $m(\cdot)$ is decreasing. Then, a firm with a better job characteristic $x_{H}$ hires a worker with lower productivity $y_{L}$, and a firm with a worse job characteristic $x_{L}\left(<x_{H}\right)$ hires a worker with higher productivity $y_{H}\left(>y_{L}\right)$. Because a worker's equilibrium utility is increasing in her productivity (see Lemma 3.1), we have $u_{H}=u\left(w\left(x_{L}\right), x_{L}\right)>u_{L}=u\left(w\left(x_{H}\right), x_{H}\right)$, where the left- 
hand side is the equilibrium utility for worker $y_{H}$ and the right-hand side is the equilibrium utility for worker $y_{L}$. The wages $w\left(x_{L}\right)$ and $w\left(x_{H}\right)$ are the lowest wages that firms $x_{L}$ and $x_{H}$ must offer to induce the worker's utility levels that are at least as high as $u_{H}$ and $u_{L}$, respectively, implying

$$
\begin{aligned}
w\left(x_{L}\right) & =\underline{w}\left(x_{L}, u_{H}\right) \\
w\left(x_{H}\right) & =\underline{w}\left(x_{H}, u_{L}\right),
\end{aligned}
$$

where $\underline{w}(x, u)$ is the lowest wage for firm $x$ to maintain the worker's utility level at $u$.

In a stable matching equilibrium, firm $x_{L}$ cannot be better off by hiring worker $y_{L}$ (instead of worker $y_{H}$ ), we have

$$
f\left(x_{L}, y_{H}\right)-w\left(x_{L}\right) \geq f\left(x_{L}, y_{L}\right)-\underline{w}\left(x_{L}, u_{L}\right)
$$

where $\underline{w}\left(x_{L}, u_{L}\right)$ is the lowest wage that firm $x_{L}$ must offer to hire worker $y_{L}$. Using Equation (6.6), we can rearrange this inequality as

$$
f\left(x_{L}, y_{H}\right)-f\left(x_{L}, y_{L}\right) \geq \underline{w}\left(x_{L}, u_{H}\right)-\underline{w}\left(x_{L}, u_{L}\right) .
$$

When the worker's utility function is supermodular, and thus, Equation (3.1) holds, Equation (6.9) implies that

$$
f\left(x_{L}, y_{H}\right)-f\left(x_{L}, y_{L}\right) \geq \underline{w}\left(x_{H}, u_{H}\right)-\underline{w}\left(x_{H}, u_{L}\right),
$$

where strict inequality holds if the worker's utility function is strictly supermodular. If the production function is supermodular, we have

$$
f\left(x_{H}, y_{H}\right)-f\left(x_{H}, y_{L}\right) \geq f\left(x_{L}, y_{H}\right)-f\left(x_{L}, y_{L}\right),
$$

with strict inequality if the production function is strictly supermodular.

If either Condition 1 or 2 in Theorem 3.1 hold, one of the two conditions (6.10) 
and (6.11) hold with strict inequality, implying that

$$
f\left(x_{H}, y_{H}\right)-f\left(x_{H}, y_{L}\right)>\underline{w}\left(x_{H}, u_{H}\right)-\underline{w}\left(x_{H}, u_{L}\right) .
$$

Using Equation (6.7), we can rearrange this inequality as

$$
f\left(x_{H}, y_{H}\right)-\underline{w}\left(x_{H}, u_{H}\right)>f\left(x_{H}, y_{L}\right)-w\left(x_{H}\right) \text {. }
$$

Note that the left-hand side of this inequality is the profit for the firm $x_{H}$ from hiring worker $y_{H}$ at wage $\underline{w}\left(x_{H}, u_{H}\right)$ and the right-hand side is the profit from hiring worker $y_{L}$. Hence, this inequality implies that there is a wage that is mutually beneficial to firm $x_{H}$ and worker $y_{H}$, which contradicts the stability of the matching function. Therefore, if the utility function is concave in consumption and either Condition 1 or 2 is satisfied, the matching function $m(\cdot)$ is an increasing function.

\subsection{Proof of Proposition 1}

Condition 1 is one of equilibrium properties that the matching and wage functions $\{m(\cdot), w(\cdot)\}$ satisfy because every firm and worker can choose to remain unmatched.

We turn to Condition 2. If the conditions stated in Theorem 3.1 are satisfied, any stable matching pattern is assortative. In other words, any non-assortative matching is not a stable matching pattern. Therefore, the assortative matching is the unique stable matching pattern given the conditions stated in Theorem 3.1 and it is characterized in Condition 2 of Proposition 1.

We prove necessity of Condition 3. Consider the firm's problem (F1). The constraint is always binding regardless of the worker whom the firm hires, because the firm's profit is decreasing in wage. Let $w^{*}(x, y)$ be the wage that makes the constraint hold with equality for all $x$ and $y$ :

$$
u\left(w^{*}(x, y), x\right)=u(w(n(y)), n(y)) .
$$


Given that the binding constraint is embedded in $w^{*}(x, y)$, this expression of wage $w^{*}$ allows us to rewrite the firm's problem as

$$
\max _{y} f(x, y)-w^{*}(x, y)
$$

The first-order necessary condition must be satisfied for all $x \geq \underline{x}$ :

$$
f_{y}(x, y)-w_{y}^{*}(x, y)=0
$$

To proceed, we derive a useful expression for $w_{y}^{*}$. Taking derivatives of both sides of Equation (6.14) with respect to $y$ yields

$$
\begin{aligned}
& \left.u_{c}\left(w^{*}(x, y), x\right)\right) w_{y}^{*}(x, y) \\
= & u_{c}(w(n(y)), n(y)) w^{\prime}(n(y)) n^{\prime}(y)+u_{x}(w(n(y)), n(y)) n^{\prime}(y) .
\end{aligned}
$$

Using the fact that $w^{*}(x, y)=w(n(y))$ and $n(y)=x$ in equilibrium, we can rewrite the equation above as

$$
w_{y}^{*}(x, y)=w^{\prime}(n(y)) n^{\prime}(y)+\frac{u_{x}(w(n(y)), n(y))}{u_{c}(w(n(y)), n(y))} n^{\prime}(y) .
$$

Substituting Equation (6.17) for $w_{y}^{*}$ in Equation (6.15), we have

$$
f_{y}(x, y)-w^{\prime}(n(y)) n^{\prime}(y)-\frac{u_{x}(w(n(y)), n(y))}{u_{c}(w(n(y)), n(y))} n^{\prime}(y)=0
$$

Given the fact that $n^{\prime}(y)=1 / m^{\prime}(n(y))$, Equation (6.18) is equivalent to Equation (3.3) in Proposition 1. Similarly, we can prove that Equation (3.3) is the first-order necessary condition for the worker.

We next prove sufficiency of Condition 3. Consider two firms $x$ and $x_{L}(x>$ $\left.x_{L} \geq \underline{x}\right)$ and two workers $y$ and $y_{L}\left(y>y_{L}\right)$. Because the sufficient conditions for assortative matching is satisfied (see Theorem 3.1), firm $x$ is matched with worker $y$, and firm $x_{L}$ is matched with worker $y_{L}$. To see if firm $x$ wants to deviate by hiring worker $y_{L}$ instead of worker $y$, we can examine the marginal changes in firm $x$ 's profit around the worker's productivity $y_{L}$. As proved in the following, they are 
given by

$$
\begin{aligned}
f_{y}\left(x, y_{L}\right)-w_{y}^{*}\left(x, y_{L}\right) & >f_{y}\left(x_{L}, y_{L}\right)-w_{y}^{*}\left(x_{L}, y_{L}\right) \\
& =0
\end{aligned}
$$

which implies that if firm $x$ currently hires a worker $y_{L}<y$, it can increase its profit by hiring someone whose productivity is slightly higher than $y_{L}$. Equality (6.20) holds because the first-order necessary condition (6.18) must be satisfied for all firms.

We begin the proof of Inequality (6.19) by showing that $w_{y}^{*}\left(x, y_{L}\right) \leq w_{y}^{*}\left(x_{L}, y_{L}\right)$. Suppose that firm $x$ hires worker $y_{L}$. Then, the derivative of the binding constraint (6.14) with respect to $y$ yields

$$
\begin{aligned}
& \left.u_{c}\left(w^{*}\left(x, y_{L}\right), x\right)\right) w_{y}^{*}\left(x, y_{L}\right) \\
= & u_{c}\left(w\left(n\left(y_{L}\right)\right), n\left(y_{L}\right)\right) w^{\prime}\left(n\left(y_{L}\right)\right) n^{\prime}\left(y_{L}\right)+u_{x}\left(w\left(n\left(y_{L}\right)\right), n\left(y_{L}\right)\right) n^{\prime}\left(y_{L}\right) .
\end{aligned}
$$

Note that Equation (6.16) at $\left(x_{L}, y_{L}\right)$ implies that the right-hand side of Equation (6.21) is equal to $\left.u_{c}\left(w^{*}\left(x_{L}, y_{L}\right), x\right)\right) w_{y}^{*}\left(x_{L}, y_{L}\right)$. Hence, we have

$$
\left.\left.u_{c}\left(w^{*}\left(x, y_{L}\right), x\right)\right) w_{y}^{*}\left(x, y_{L}\right)=u_{c}\left(w^{*}\left(x_{L}, y_{L}\right), x_{L}\right)\right) w_{y}^{*}\left(x_{L}, y_{L}\right)
$$

Observe that firm $x$ 's wage offer $w^{*}\left(x, y_{L}\right)$ that induces the utility level $u(w(n(y)), n(y))$ is lower than $w^{*}\left(x_{L}, y_{L}\right)$, i.e., $w^{*}\left(x, y_{L}\right)<w^{*}\left(x_{L}, y_{L}\right)$, because the utility monotonically increases with job characteristic and $x>x_{L}$. Because the utility function is concave in consumption and supermodular, the fact that $w^{*}\left(x, y_{L}\right)<w^{*}\left(x_{L}, y_{L}\right)$ and $x>x_{L}$ implies

$$
\left.\left.u_{c}\left(w^{*}\left(x, y_{L}\right), x\right)\right) \geq u_{c}\left(w^{*}\left(x_{L}, y_{L}\right), x_{L}\right)\right) .
$$

Given this inequality, for Equation (6.22) to hold, it must be true that

$$
w_{y}^{*}\left(x, y_{L}\right) \leq w_{y}^{*}\left(x_{L}, y_{L}\right)
$$


If the utility function is supermodular and the production function is strictly supermodular (i.e., Condition 1 of Theorem 3.1 holds), we have Equation (6.24) and $f_{y}\left(x, y_{L}\right)>f_{y}\left(x_{L}, y_{L}\right)$, implying Inequality (6.19) holds. If, instead, the utility function is strictly supermodular and the production function is supermodular (i.e., Condition 3 of Theorem 3.1 holds), Equation (6.24) holds with strict inequality and we have $f_{y}\left(x, y_{L}\right) \geq f_{y}\left(x_{L}, y_{L}\right)$, implying Inequality (6.19) holds.

In the discussion above, we prove that hiring a worker $y_{L}<y$ is less profitable for the firm $x$ than hiring the worker $y$. Next, consider if it is profitable for firm $x$ to hire worker $y_{H}>y$. By the argument similar to the one above, we can also show that

$$
f_{y}\left(x, y_{H}\right)-w_{y}^{*}\left(x, y_{H}\right)<0
$$

for all $y_{H}>y$. This shows that if firm $x$ currently hires worker $y_{H}>y$, it can increase its profit by hiring someone whose productivity is slightly lower than $y_{H}$. Therefore, firm $x$ can maximize its profit by hiring the worker whose productivity is $y=m(x)$ under the assortative matching function $m(\cdot)$. We can similarly prove that Equation (3.3) is also the sufficient condition for the worker's problem (W1). 